\title{
ESCOLARIZACIÓN EN CONFINAMIENTO: EXPERIMENTO NATURAL Y PRUEBA DE ESFUERZO
}

\author{
Schooling in Confinement: Natural Experiment and Stress Test
}

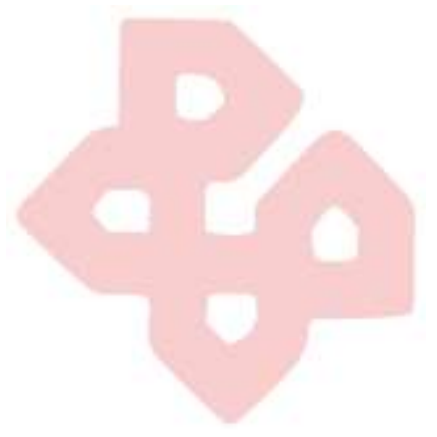

\author{
Juan Manuel Moreno y Lucas Gortazar ${ }^{1}$ \\ Banco Mundial/The World Bank \\ E-mail: jmoreno@worldbank.org; \\ lgortazar@worldbank.org \\ ORCID ID: https://orcid.org/0000-0002-3029-771X \\ https://orcid.org/0000-0002-6785-1059
}

Resumen:

La pandemia causada por el coronavirus en $\mathbf{2 0 2 0}$ ha supuesto un auténtico experimento natural que permite obtener evidencia empírica sobre el contrafactual de "qué pasaría si las escuelas cerraran"; y al mismo tiempo, ha sido una prueba de esfuerzo en toda regla que ha puesto transparentemente de manifiesto tanto las fortalezas como las debilidades del sistema educativo en su conjunto y las de todos y cada uno de sus actores y componentes. Nuestra posición de partida es que las crisis creadas por la pandemia están acelerando tendencias y procesos, desafíos y problemas, y que tal aceleración de la Historia supone tanto nuevos riesgos como nuevas oportunidades para los sistemas escolares. Este artículo pretende explorar, a partir de la evidencia empírica disponible, esos riesgos y oportunidades y qué opciones existen para proteger y sostener el sistema escolar público en la nueva normalidad.

\footnotetext{
1 Juan Manuel Moreno y Lucas Gortazar son, respectivamente, Especialista Principal de Educación y Analista del Banco Mundial. Las ideas y opiniones planteadas en este artículo son exclusivamente de los autores y no representan la posición oficial del Banco Mundial.
} 
Escolarización en Confinamiento: Experimento natural y prueba de esfuerzo

Palabras clave: autonomía escolar; brechas digitales; cierre de escuelas; desigualdad; liderazgo profesional del profesorado.

\section{Abstract:}

The COVID-19 pandemic entailed a true natural experiment that allows to collect empirical evidence about the counterfactual of "what would happen if all school closed"; and at the same time, it has amounted to a thorough stress test that has transparently displayed both the strengths and weaknesses of education systems as a whole and of each and every actor and element within them. Our starting point is that the crises created by the pandemic have accelerated trends and processes, challenges and problems, and that such acceleration of History implies both new risks and opportunities for school systems. This paper uses some of the existing empirical evidence to explore those risks and opportunities and the options available to protect and sustain public school systems under the new normal.

Key words: digital gaps; inequality; school autonomy; school closures; teachers' professional leadership.

\section{Introducción}

En educación, la crisis del Covid19 se resume en el cierre de centros educativos desde mediados de marzo de 2020 y en los planes para una fecha de reapertura en septiembre. Esta situación claramente distópica ha supuesto, por una parte, un auténtico experimento natural que permite obtener evidencia empírica sobre el contrafactual de "qué pasaría si las escuelas cerraran"; y por otra, una prueba de esfuerzo en toda regla que ha puesto transparentemente de manifiesto todas las debilidades y fortalezas del sistema educativo en su conjunto y de todos y cada uno de sus actores y componentes.

La pandemia causada por el COVID-19 y sus crisis resultantes - sanitaria, económica y educativa - han creado un nuevo escenario político y están rediseñando el tablero en el que los distintos jugadores mueven sus fichas. Hay quienes están convencidos de que el juego nunca volverá a ser igual, que para bien y para mal todo será distinto y se regirá por nuevas reglas; y al otro lado, hay quienes no tienen duda de que todo volverá a ser como antes y que la nueva normalidad terminará pareciéndose a la vieja como una gota de agua se parece a otra. Entre esas dos posiciones extremas, abrumadoramente mayoritarias si sumamos sus seguidores en este momento, caben posiciones intermedias, que es donde se sitúa este artículo. Nuestra posición de partida es que las crisis en cuestión están acelerando tendencias y procesos, desafíos y problemas, y que tal aceleración de la Historia supone tanto nuevos riesgos como nuevas oportunidades para los sistemas escolares. Existen además datos que así lo que corroboran.

Aparece aquí con prominencia la cuestión del liderazgo, entendido como la combinación de visión política con iniciativa profesional para materializarla, todo ello en el contexto de instituciones educativas más o menos sólidas. Si la pandemia es una prueba de esfuerzo para todo y para todos, lo está siendo de un modo especialmente 
agudo para los políticos y gestores, y para las instituciones de las que son responsables. También nuestros líderes - políticos y profesionales de la educación - están siendo sometidos a la prueba de sus vidas; si su posición lleva aparejado el coste de tomar decisiones difíciles, con esta crisis se están viendo confrontados con encrucijadas en las que todas las opciones son malas política y profesionalmente hablando, es decir, todas conllevan un enorme esfuerzo y riesgo y todas implican ver crecer el número de sus enemigos. Así las cosas, si la calidad del liderazgo político y profesional en educación ya era baja, y sus instituciones frágiles, no debe sorprender que ahora lo esté siendo más todavía, y que ello redunde en debilitar todavía más al sector y en que aquellos que solo defienden los intereses de parte se crezcan ante quienes tienen la responsabilidad de defender los intereses de todos, pero no la capacidad e influencia necesarias para hacerlo.

Este articulo pretende explorar, a partir de la evidencia empírica disponible, los riesgos y oportunidades para la escuela causados por la pandemia, y qué opciones existen para proteger y sostener el sistema escolar público en la nueva normalidad.

\section{Experimento natural y prueba de esfuerzo}

1. Se ha puesto de manifiesto el valor añadido de la escolarización al poder medir las consecuencias de su cierre en prácticamente todos los países del mundo y por una duración mayor que cualquier periodo vacacional. La conclusión es que, además de haberse producido una pérdida de aprendizaje tan aguda como generalizada, la presencialidad en la enseñanza y el aprendizaje es insustituible, y que la función de la escolarización como espacio de socialización y de custodia de niños y adolescentes es crucial. Las primeras evidencias de métricas de aprendizaje durante el confinamiento muestran que el ritmo de aprendizaje se ha deteriorado, especialmente para los más vulnerables. En el caso de España, Bonal y González (2020) muestran que, durante las primeras semanas del confinamiento, un porcentaje importante de alumnos $(28,3 \%)$ prácticamente no tuvo acceso a ninguna actividad de aprendizaje ni relación virtual con docentes o tutores: esto podría tener consecuencias dramáticas si esa desconexión virtual se convierte en absentismo y abandono reales cuando se retome la presencialidad, y ello tanto en países desarrollados como, sobre todo, en países en desarrollo. Además, se dan importantes brechas de acceso al indicador de "Oportunidades de Aprendizaje" construido por los autores: llama especialmente la atención que sean más importantes las diferencias por titularidad de centro (reflejando mucha más actividad virtual en centros privados y concertados que en públicos) que por renta de las familias (Gráfico 1). Datos semejantes de brechas de actividad, comunicación y aprendizaje a distancia se han encontrado en estudios llevados a cabo en otros países, como por ejemplo Holanda y Reino Unido (Andrew et al, 2020; Bol, 2020). 
Por encima incluso de la evidencia sobre pérdidas masivas de aprendizaje, lo que queda claro en este análisis contrafactual al que nos hemos visto forzados es que el cierre de escuelas tiene efectos inmediatos sobre la cohesión social y la equidad, es decir, sobre la inclusión social, cultural y laboral de los ciudadanos. La escolarización como espacio público y como servicio público permite acceder y luego formar parte activa de otros muchos espacios e instituciones; y en este sentido, se ve con claridad que el cierre de ese espacio público tiene un efecto devastador sobre los estudiantes más desfavorecidos socioeconómicamente.

Figura 1. Oportunidades de Aprendizaje, nivel socioeconómico y titularidad de centro.

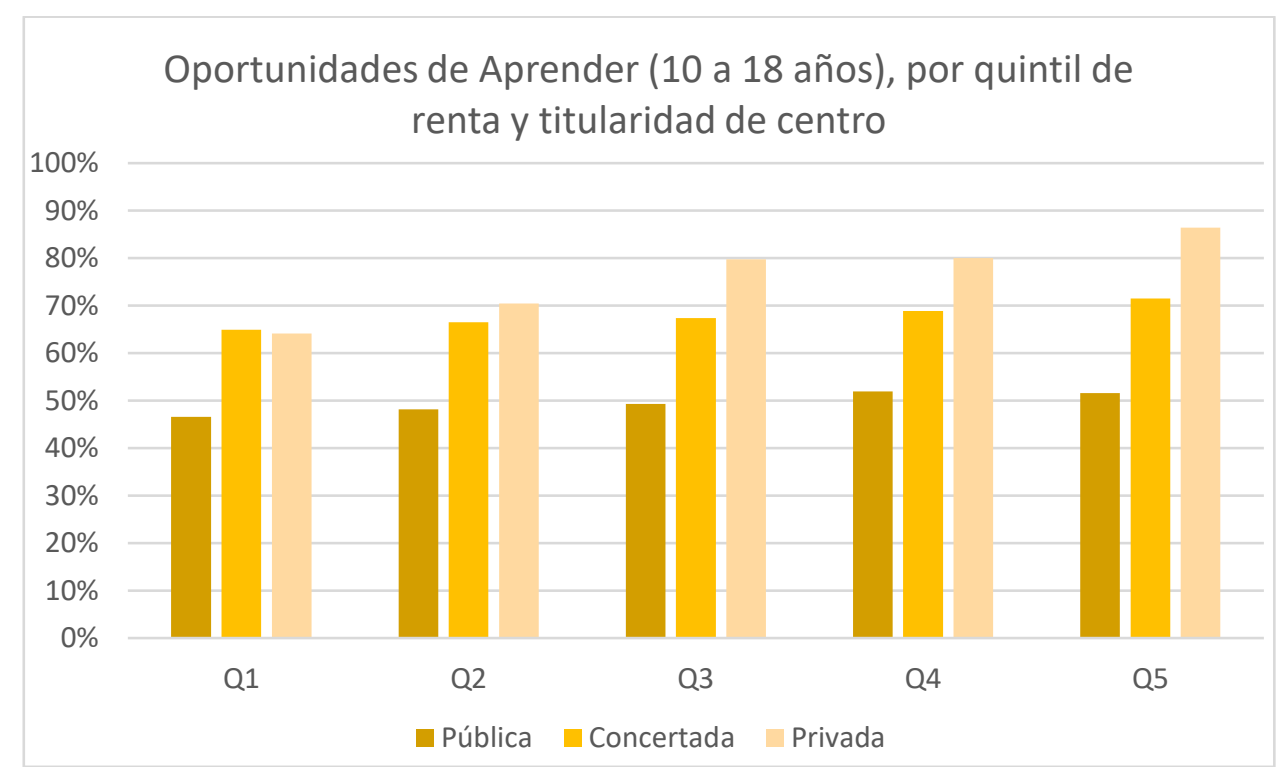

Fuente: Bonal y Gonzalez (2020). El índice (OTL, Opportunities to Learn), está construido a partir de las variables del cuestionario referidas al trabajo escolar durante el confinamiento, a la comunicación online con el profesorado, a la recepción de mails, al encargo de tareas para realizar en solitario y a la realización de trabajos para ser corregidos. El índice oscila entre 0 y 100 , donde 0 equivale a ausencia de OTL y 100 equivale a un OTL máximo.

2. La digitalización del sector educativo ha permitido que las escuelas siguieran abiertas aun estando cerradas. La pérdida de aprendizaje hubiera sido mucho mayor de no ser por la movilización de soluciones tecnológicas que todos los países han realizado. En este sentido, queda pendiente estimar el grado en que se ha acelerado el proceso de digitalización de la educación, uno de esos procesos que ya estaba en marcha y que la respuesta a la pandemia ha impulsado con fuerza. Por esta razón, los planes de reapertura de los centros y los de contingencia para preparar al sistema educativo ante posibles rebrotes en el futuro tienen como pilar fundamental la consolidación de la digitalización. Esto podria conducir, a su vez, a cambios acelerados en la concepción de los espacios escolares, la configuración de horarios, y el uso que hagan de tiempo y espacio escolares tanto docentes como estudiantes.

3. Los problemas de equidad y de segregación educativa han salido del interior de las aulas y se han hecho visibles más allá del sector. A la segregación socioeconómica que ya encontramos entre escuelas de muchos 
sistemas educativos se suma el hecho de que cada alumno de una misma escuela pase a estar confinado en su propio hogar. Los datos sobre cómo el propio esfuerzo tecnológico y digital contribuye a exacerbar aún más las desigualdades de acceso a la educación, de uso de tecnología para el aprendizaje, y de capacidad institucional de centros y profesorado para operar durante los cierres (y después en la nueva normalidad), ponen de manifiesto que la inversión en digitalización es condición necesaria pero no suficiente para garantizar el derecho a la educación y la continuidad en el aprendizaje de todos los estudiantes en unas condiciones de igualdad efectiva de oportunidades. Los datos de PISA 2018 muestran que, en todos los sistemas educativos del mundo desarrollado, entre un $20 \%$ y un $60 \%$ de los centros educativos no estaban capacitados para operar durante los cierres (Gráfico 2). El propio modelo y la manera de articular el desarrollo de la digitalización en educación va a determinar la evolución de esa triple dimensión de la brecha digital, que podría seguir ampliándose incluso después - y a pesar - de fuertes inversiones en conectividad y equipamiento.

Figura 2. Proporción de directores de centro que están de acuerdo con la afirmación "Una plataforma online eficaz para apoyar el aprendizaje está disponible en el centro"

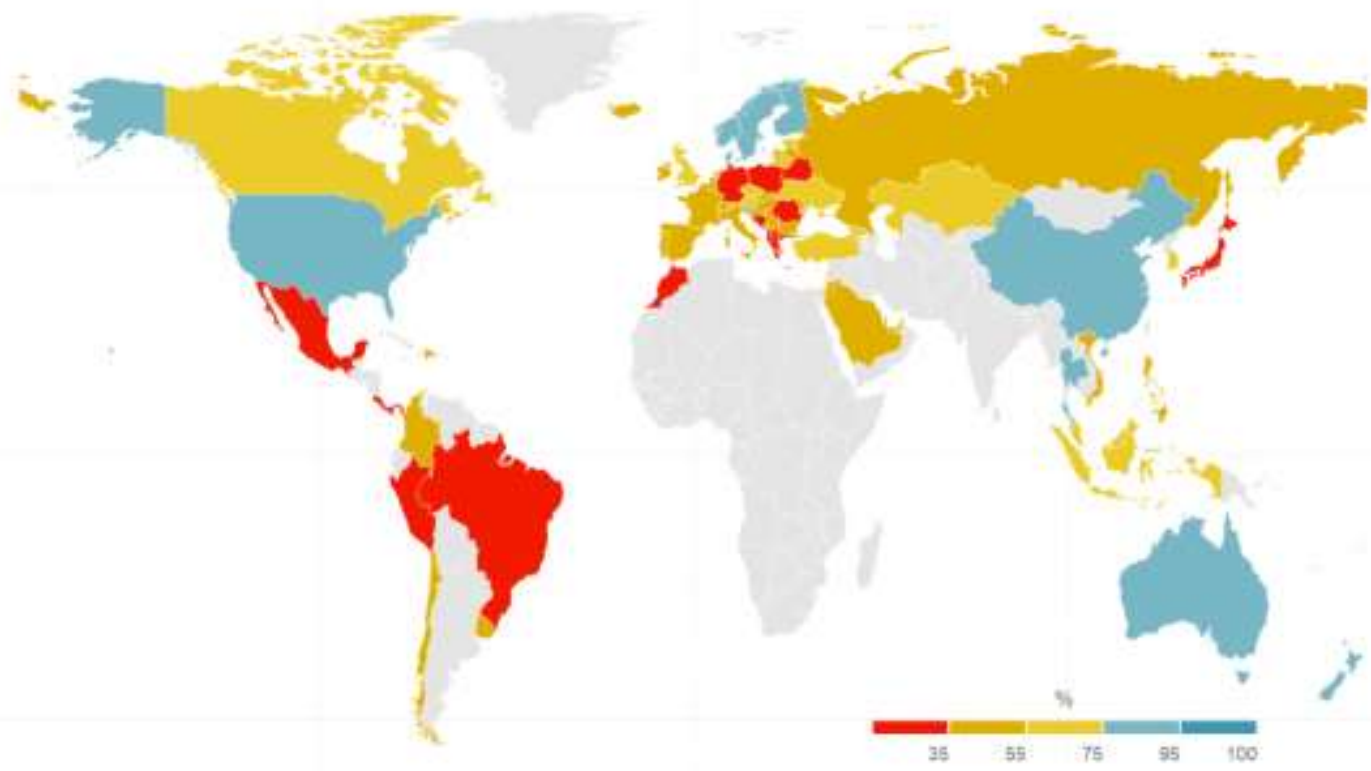

Fuente: Moreno y Gortazar (2020).

4. Está cambiando la percepción pública sobre la escolarización que tenían antes de la crisis tanto los miembros de la comunidad educativa profesorado y familias principalmente - como los empleadores (públicos y privados), los medios de comunicación, los partidos políticos y la opinión pública en general. Las familias tienen hoy una experiencia directa sobre el trabajo académico de sus hijos que no habían tenido antes con esta intensidad. 
Esta nueva conciencia pública sobre la escolarización crea la oportunidad de elevar el nivel del debate educativo en cada país, de aprender sobre lo que han hecho otros sistemas educativos y aprovechar su experiencia e innovaciones como un bien público global; también ayuda a fortalecer el consenso para que la inversión en educación se mantenga como pilar fundamental de la inversión en supervivencia en la que el mundo está volcado (Moreno, 2020).

Por otra parte, es previsible que las familias aprovechen su experiencia directa y mayor conocimiento de la escolarización para consolidar una mayor implicación en las decisiones de cariz más académico, incluida la evaluación, relacionadas con sus hijos. Esto, que sin duda es una buena noticia (la evidencia empírica sobre la relación entre los niveles de implicación de las familias y rendimiento académico de los estudiantes es abrumadora ${ }^{2}$ ), puede percibirse como una amenaza por parte de algunos centros y profesores. Pero el cierre de centros y el confinamiento han abierto varias cajas de Pandora y parecería más inteligente aprovechar las oportunidades que ello crea que tratar de cerrar esas cajas de nuevo.

5. Al responder a la crisis creada por la pandemia, la cuestión de la autonomía escolar surge como el tema central de la gobernanza de los sistemas educativos. Cuando se trata de dar respuestas rápidas a una situación de emergencia, las administraciones y su modelo habitual de gestión lo han hecho mediante una provisión de servicios lenta, a menudo totalmente centralizada, poco conectada con la información que dispone cada centro sobre sus realidades y necesidades, y basada en el control burocrático de procesos. Por su parte, los centros, que en muchos sistemas educativos tienen escasos hábito y capacidad de tomar decisiones estratégicas complejas que obliguen a gestionar los recursos de la manera más eficiente posible, se topan con una realidad que en muchos casos no saben afrontar. En este contexto, se han impuesto el miedo y la aversión al riesgo inherente a tomar cualquier decisión, lo que crea una barrera más para garantizar la continuidad en el aprendizaje de los estudiantes, al tiempo que ahonda la brecha entre escuelas públicas y privadas e incluso entre escuelas del mismo sector. Y, por tanto, la misma autonomía que sirve a algunos centros para diseñar y poner en marcha una respuesta innovadora y bien ajustada a su realidad se convierte para otros centros, de facto, en aislamiento y soledad institucional.

6. Quién y qué es esencial dentro del sistema educativo está siendo fuertemente cuestionado en el debate sobre el regreso a los centros. Mientras que la reapertura de espacios públicos y sectores económicos ha ido avanzando decididamente en todo el mundo con relativamente poca polémica a pesar de los indudables riesgos de salud, la reapertura de los centros educativos está siendo mucho más controvertida y mucho más lenta, además de haber dado lugar a un debate más agrio. El Grafico 3 muestra la evolución del porcentaje de estudiantes de todo el mundo afectados por el cierre de escuelas entre marzo y junio de 2020. En esta última fecha, cuando son muy

\footnotetext{
${ }^{2}$ Bruns, Filmer y Patrinos (2011).
} 
pocos los países que mantienen medidas de confinamiento de la población, casi el 80 por ciento de los estudiantes a escala mundial seguía sin poder acceder a su escuela.

Figura 3. Distribución de los estudiantes por situación de escolarización en todo el mundo, entre marzo y junio de 2020.

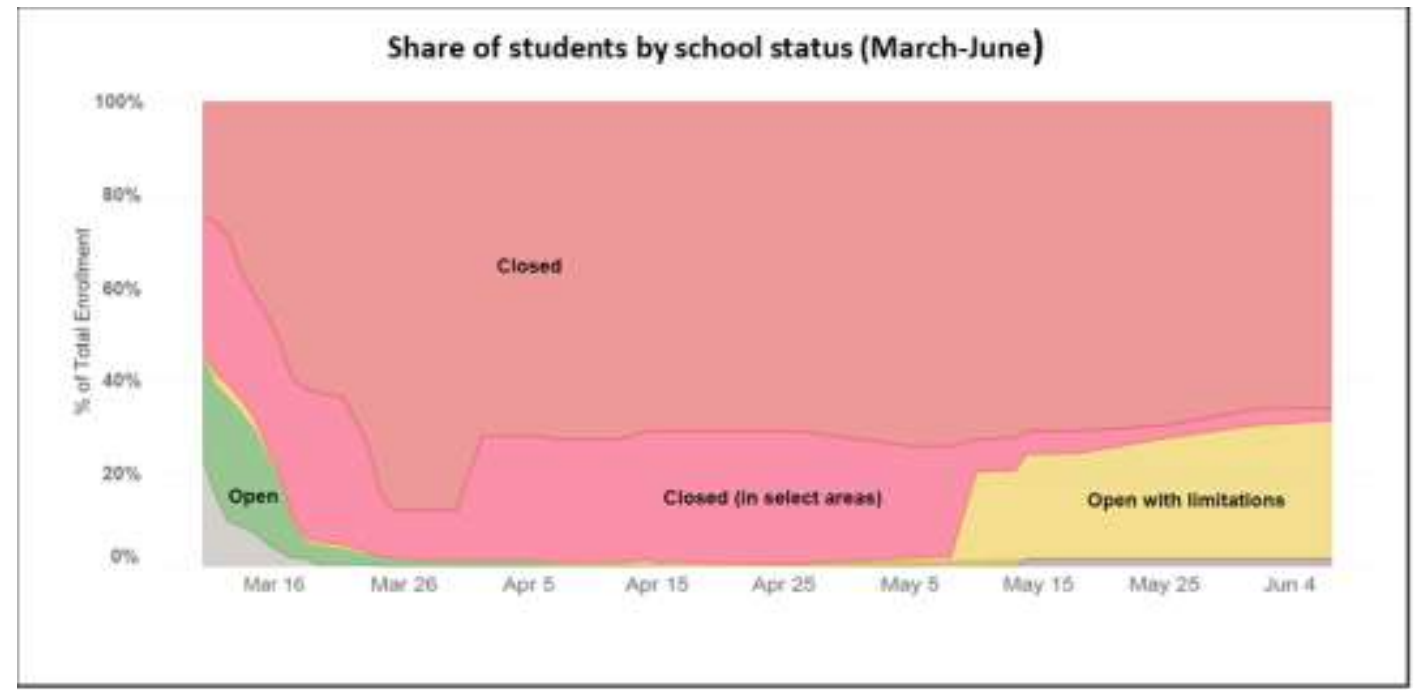

Fuente: Banco Mundial (2020).

En el escenario de la paulatina reapertura de los centros, los intereses de algunos profesores (suelen ser, por razones obvias, los más afectados por la falta de inversión) y de los sindicatos docentes se contraponen a los de los estudiantes. Además, los intereses de la economía productiva, es decir, los de toda la ciudadanía, van en este caso de la mano de las familias (y de los alumnos). Quizás ésta sea la manifestación más clara que jamás se haya visto de la tensión o incluso del conflicto latente entre el derecho a la educación de los niños y niñas (que familias, administraciones y tejido productivo exigen) y el derecho del profesorado a su seguridad laboral.

Abrir los centros es una de las medidas fundamentales para salir de la crisis y, aunque quizás haya de ser la última, es sin duda la definitiva, ya que, por ejemplo, en el caso de España, podría afectar al $35 \%$ de la oferta trabajo total ${ }^{3}$. Por tanto, desde un punto de vista económico, social y educativo, merece la pena hacer "todo lo que sea posible" por parte de los gobiernos; y que ello, por completar la ya legendaria cita de Mario Draghi, "sea suficiente".

\footnotetext{
${ }^{3}$ Con datos de la Encuesta de Condiciones de Vida (2018) para el caso de España, la población de 25 a 64 años con menores de 16 años a cargo representa un 36.8\%. Considerando que la población española es, junto con la italiana, una de las más envejecidas del mundo, este porcentaje será necesariamente más alto en el resto del mundo.
} 
3. Tendencias y procesos que se han acelerado y problemas y riesgos futuros que se agudizan como resultado de la crisis del Covid-19

La demanda de educación se ha mantenido firme $e$ incluso se ha incrementado. Esto podría cambiar radicalmente si el cierre de centros se alargara mucho o si la pandemia tuviera brotes sucesivos a lo largo de años que obligaran a nuevos periodos de confinamiento. La desaparición progresiva del espacio público de la escolarización no solo elevaría la tasa de abandono virtual, sino que también incrementaría la de abandono real o definitivo, además de los niveles de desafección escolar por parte de los estudiantes y de sus familias. Además, en muchos países en desarrollo que aún tienen un número considerable de estudiantes que nunca han estado escolarizados, sería imposible reducirlo a corto plazo, como se esperaba antes de la pandemia, e incluso podrían ver ese número incrementado a medio y largo plazo.

La inversión privada de las familias en educación sigue subiendo. Esto era inevitable en el contexto del confinamiento, porque de entrada las familias han tenido que crear una escuela en casa, poniendo a disposición de sus hijos ordenadores, teléfonos, conexión a internet y, lo más importante, tiempo para supervisar y apoyar su trabajo escolar. Quienes han podido, han aumentado la inversión en clases particulares, aunque fueran también virtuales. Con la crisis económica, esta tendencia podría cambiar radicalmente si, con tasas de desempleo al alza y pérdidas de renta generalizadas, las familias se vieran forzadas a recortar gastos directamente relacionados con la educación de sus hijos (Martínez-García y Molina, 2019).

La brecha entre escuela pública y privada se sigue extendiendo. Si las escuelas privadas (sean o no financiadas con fondos públicos) han gozado en las últimas décadas de mayor autonomía para hacer frente a las decisiones organizativas y de gestión de recursos, cabe pensar que estaban más habituadas y preparadas para dar una respuesta ágil y eficaz a la crisis generada por la pandemia, y es igualmente lógico asumir que estarán más preparadas para el regreso a la presencialidad bajo las actuales condiciones y restricciones sanitarias. Esa ventaja puede agrandarse en los próximos cursos a los ojos de las familias y del público en general, ahondándose con ello las brechas público-privadas ya existentes. Sin embargo, la crisis económica que viene como secuela inmediata de la crisis sanitaria daría la vuelta a esta tendencia si muchas familias no fueran capaces de mantener a sus hijos en la enseñanza privada. De hecho, muchas escuelas privadas se verían obligadas a cerrar en el contexto de la crisis económica que parece inevitable; un ejemplo ya bien conocido es el de las escuelas privadas de bajo coste en muchos países en desarrollo (Banco Mundial, 2020). La brecha de resultados entre pública y privada seguiría existiendo, pero el volumen del sector privado podría reducirse dramáticamente y el sector público verse por consiguiente obligado a hacerse cargo de esos nuevos estudiantes, lo que a su vez le dejaría menor margen de maniobra y menos recursos para recuperar a los estudiantes más vulnerables y compensar las enormes pérdidas de aprendizaje que han sufrido.

El factor escuela y el liderazgo profesional del profesorado marcan la diferencia ahora más que nunca. La crisis del coronavirus ha demostrado - y no solo en el sector educativo - que los funcionarios que siguen haciendo su trabajo y asumen 
su responsabilidad, todo ello sin hacer ruido ni ponerse medallas, son los que salvan al sistema. La lección, tal vez más importante, que se ha aprendido hasta el momento es que la gran diferencia, en cuanto al objetivo de proteger el aprendizaje de los estudiantes, la están marcando los centros escolares donde existía ya una tradición fuerte de trabajo colaborativo y de innovación entre el profesorado (que permitía una mejor organización del tiempo de contacto y comunicación con los alumnos y, paradójicamente, un menor tiempo de dedicación), un liderazgo sólido y una capacidad fuerte para movilizarse y encontrar soluciones viables para mantener a los estudiantes involucrados en la escuela y en su propio aprendizaje.

No nos ha sorprendido que, sin escuela, la experiencia de cada alumno dependa mucho de su hogar; y no debería sorprendernos que, sin carrera profesional docente, el desempeño y la experiencia de cada docente durante el confinamiento dependa mucho de su capacidad y voluntad individual, que por definición es tan dispar como la de los alumnos. Del mismo modo que tenemos un sistema educativo como servicio público para garantizar experiencias semejantes de aprendizaje y socialización de los alumnos, se debe aspirar a tener un sistema de desarrollo profesional que garantice una preparación, apoyo y oportunidades de carrera profesional iguales para todos los docentes.

Podríamos estar en el principio del fin del papel central y hegemónico de los libros de texto en la configuración del currículo en la práctica. Los meses de escolarización en confinamiento han puesto de manifiesto que el currículo escolar está cada vez menos en los libros de texto y cada vez más en las plataformas digitales públicas y privadas. Esto abre una posibilidad real de evolucionar hacia modelos curriculares centrados en la adquisición de competencias, una tendencia que ya era fuerte en materia de política curricular mucho antes de que irrumpiera la crisis sanitaria del coronavirus. En concreto, como apunta Pritchett, usando la evidencia disponible (2020), los ajustes curriculares diseñados para compensar los efectos del cierre de centros y que además busquen adaptarse mejor a los niveles de competencia de los estudiantes más afectados pueden contribuir de un modo notable, a medio plazo, a mitigar el impacto del confinamiento sobre la pérdida de aprendizaje de los alumnos

Por una parte, es previsible un aumento del estudio independiente del alumnado más allá del periodo de confinamiento. Ya no cabe duda de que los modelos mixtos de escolarización que se vislumbran en la reapertura implican un mayor peso del trabajo y estudio independiente de los estudiantes. Por otra parte, la rigidez y la inercia históricas de las materias académicas, los horarios y tareas escolares han saltado por los aires con esta crisis. Muchos centros y docentes han descubierto por la via de los hechos que se puede trabajar de un modo más flexible y dinámico, tanto en lo que se refiere a los contenidos curriculares como a los tiempos asignados a tareas y proyectos. Son previsibles una tendencia fuerte a restaurar el régimen tradicional y al mismo tiempo otra que continúe explorando las posibilidades que se han abierto. Una vez más, aquí puede identificarse una brecha creciente de innovación curricular entre centros, sean públicos o privados. 
La evaluación del rendimiento estudiantil en el contexto del aprendizaje a distancia continúa sin resolverse en la nueva normalidad. No estaba bien resuelta ya antes de la pandemia, y ahora lo está todavía menos y se enfrenta a problemas graves: cronificación de la repetición y del abandono en los países sin cintura reguladora para hacer cambios; dificultades logísticas y técnicas para realizar exámenes a distancia y generalización de las prácticas corruptas en este ámbito fraude, copia, suplantación de personalidad, compra de exámenes y trabajos académicos en la red, etc. Por el contrario, en aquellos países donde se han tomado medidas para prevenir que la brecha digital cause más fracaso, repetición y abandono, se podrían haber puesto los cimientos para erradicar esos problemas en el futuro. Sabemos que, en esencia, fracaso, repetición y abandono se deben a una inercia cultural que es interna a los sistemas escolares y que esta crisis podría haber "sacudido" y hecho evolucionar.

El concepto de escuelas seguras va a cobrar un sentido nuevo en el corto plazo, y quizás también en el medio y largo. El concepto de escuelas seguras se había abierto paso con fuerza en la última década, sobre todo en relación con la prevención de la violencia escolar (desde el bullying hasta la violencia escolar de género). Ahora el concepto se expande notablemente para incorporar la seguridad sanitaria. La nueva normalidad escolar tiene un fuerte potencial para que avance la agenda de escuelas seguras desde el punto de vista de la prevención de la violencia y la promoción de la convivencia democrática. Por otro lado, surgirá con más fuerza la preocupación por la seguridad digital de los estudiantes y será necesario invertir en ella tanto como en la seguridad sanitaria.

\section{Escenarios para la Nueva Normalidad: El Feo, el Malo y el Bueno}

\section{Escenario 1: Hundimiento}

Se trata de un escenario apocalíptico para los sistemas escolares. Según este planteamiento, el efecto dominó que lleva desde la crisis sanitaria a la escolar, pasando por una devastadora crisis económica y del empleo, pondría la escuela pública en peligro de desaparición definitiva y, en el proceso que iría hasta la culminación de la catástrofe, irían aumentando las desigualdades de clase, de género, territoriales, etc. La educación seguiría existiendo, pero sería cada vez menos escolar y sobre todo menos pública. Desde esta posición, se entiende que solo doblar o triplicar de manera urgente la contratación de profesorado y hacer lo propio con la inversión en infraestructuras físicas y tecnológicas podría detener el apocalipsis de la escuela como institución.

Sin embargo, como sugerimos más arriba, la economía solo podrá reabrirse y relanzarse del todo cuando el sistema escolar reabra por completo, y esto se añade a la renovada percepción del valor añadido de la escolarización para el conjunto de la sociedad civil y para los principales actores políticos. Además, el riesgo de cierres definitivos y aun de desaparición absoluta de centros está mucho más en los niveles no 
obligatorios del sistema, es decir, en la universidad y la educación infantil, donde por cierto el sector privado tiene mayor peso y en muchos países es incluso mayoritario. Por tanto, hay mucho más riesgo de que desaparezca la educación privada que la pública y, en todo caso, es mucho más probable que disminuya el peso de la educación privada que el de la pública (en términos de tasas de matrícula). El caso de las universidades privadas en todo el mundo es alarmante, sobre todo por el retroceso en la democratización de la educación superior que ello va a suponer y por la pérdida de millones de puestos de trabajo de académicos e investigadores jóvenes y con contratos precarios en esas instituciones privadas. Algunos podrían leer todo esto como una repercusión positiva de la pandemia, pero no lo es en absoluto, por más que en ambos sectores - infantil y superior - pueda servir para sacar del mercado a proveedores de muy baja calidad. Si el peso de la privada disminuye, como también se ha apuntado anteriormente, el sector público tendrá que hacerse cargo de esos estudiantes, y eso en un contexto de recortes presupuestarios y de restricciones de espacio, equipamiento, infraestructura y, sobre todo, profesorado.

\section{Escenario 2: Reiniciar el Sistema y Escuela 2.0}

Este es el escenario del optimismo histórico que, mediante la superación de conflictos de forma eficiente, nos llevaría a las más altas cotas de progreso. De igual modo que, cuando se bloquea un ordenador o un teléfono, la mejor opción es apagarlo y 'reiniciar', son muchos los que creen - o al menos esperan - que la pandemia ha creado la oportunidad histórica de apagar y volver a encender el sistema escolar, esperando que lo que lo bloqueaba, o todo lo que lo hacía funcionar lento y mal, haya desaparecido por el efecto mágico-benéfico del reseteo. Tendríamos pues la oportunidad de poner en marcha un sistema educativo 2.0 gracias al principio de la disrupción positiva o, si se prefiere, de destrucción creativa, a lo Schumpeter. Un optimismo histórico que insiste en que saldríamos fortalecidos de la catástrofe y además con los problemas endémicos del sector resueltos dadas las oportunidades que crea el tránsito a la nueva normalidad. En algún caso, este optimismo histórico no se manifiesta solo como esperanza, sino que se esgrime como una exigencia a la que los sistemas escolares, los centros y el profesorado deben responder. Estos últimos habrían pasado, en muy poco tiempo, de verse desafiados a ejercer su trabajo en condiciones totalmente sin precedentes, a verse presionados para asumir cambios y reformas radicales que habrían de ejecutarse casi de un día para otro. Se pasa por alto, entre tanto optimismo militante, que el cierre de centros no se ha llevado a cabo deliberadamente como medida mágico-benéfica para hacer posible la gran revolución educativa, sino que el cierre ha interrumpido un servicio público que, a su vez, garantiza un derecho humano, y que la prioridad es recuperar ambos, el servicio y el derecho, de un modo sostenible ante las nuevas circunstancias.

Es posible pues que este optimismo histórico ignore que la escuela pública (y privada) se mueve siempre entre coaliciones de intereses por parte de los distintos actores, y más ahora que la tensión entre el derecho a la educación de los alumnos y el derecho a proteger la seguridad laboral (y sanitaria) del profesorado está más presente que nunca. A su vez, asumiendo a muy corto plazo un contexto de restricción 
presupuestaria o incluso recortes, es improbable que se produzca una fuerte inversión pública en la educación durante los próximos años, lo que obviamente va a dificultar el apoyo a los procesos de cambio por parte de los profesionales de la educación; al contrario, en este contexto, lo probable es que la prioridad de los gobiernos sea reforzar la salud pública (que es la gran ganadora entre los pilares del estado de bienestar como resultado de la pandemia), seguida de las políticas de protección social, además de la garantía de sostenibilidad de las pensiones.

\section{Escenario 3: Rebote versus Rebrote}

Es ya tristemente evidente que los cierres de escuelas van a suponer una regresión notable en la evolución de la brecha de aprendizaje entre los estudiantes de todo el mundo. Los más desfavorecidos, incluso en los países más ricos, van a sufrir una desventaja todavía mayor, con la consiguiente disminución de oportunidad de aprender, de años de escolarización y de perspectivas de inclusión social y laboral. Dicha brecha se había ido cerrando en muchos países, especialmente desde 2012, al hilo de la disminución de los niveles de pobreza y de pobreza extrema en todo el planeta. Con esta crisis, esas perspectivas tan positivas deben, por desgracia, abandonarse. Los estudios que ya se han llevado a cabo en países como el Reino Unido (Andrew et al, 2020) estiman que la brecha de desigualdad ha crecido solo en estos meses entre el 11 y el 75 por ciento. Hay por tanto incertidumbre incluso en cuanto a cuál podría ser la magnitud del problema al que se enfrentan los sistemas escolares.

No obstante, si no hay rebrote del virus, puede haber rebote educativo y de aprendizaje, es decir, del mismo modo que los más optimistas hablan de una recuperación en $\mathrm{V}$ de la economía (o en forma del logo de Nike los optimistas moderados), podría haber un rebote similar en la pérdida de aprendizaje si se ponen en marcha medidas agresivas de rescate del sistema educativo. Estas medidas irían desde programas de tutoría individualizada a alumnos con mayor desventaja para recuperar el aprendizaje perdido hasta becas-salario como incentivos económicos para evitar el surgimiento de una generación de alumnos absentistas provenientes de familias en riesgo de exclusión social. Si hubiera rebrote viral, sin embargo, los riesgos se multiplicarían, y la caída de las constantes vitales del sistema escolar podria profundizarse y tener consecuencias a muy largo plazo. La sociedad podria verse obligada a buscar otras alternativas ya muy distintas a la escolarización tradicional, que irremediablemente supondrían, como mínimo al comienzo de ese proceso, una profundización de la desigualdad como gran desafío global.

\section{Conclusión}

Es urgente fortalecer tanto el liderazgo profesional del sector educativo como las instituciones del sistema escolar, empezando obviamente por cada escuela, y desarrollarlos en espacios amplios de consenso y participación de distintos actores. La autonomía escolar tiene que habilitarse no solo desde y para la gestión de recursos humanos y presupuestarios sino también para la generación de instituciones resilientes 
ante crisis como la vivida en 2020. Sea cual sea el escenario que nos resulte más realista, la capacidad de cada centro educativo para reorganizarse y del liderazgo profesional de los educadores para buscar soluciones adaptadas son la única esperanza de que las instituciones educativas no colapsen ante los riesgos que tienen por delante.

Es tiempo por tanto de que los poderes públicos y la sociedad en general apuesten por el rebote educativo para que el sistema escolar se consolide como pilar de la recuperación cultural y económica de nuestras sociedades. Desde los poderes públicos, la crisis podría estar creando un espacio político amplio para impulsar una segunda generación de transformaciones educativas que redunden en la profesionalización docente y en asegurar confianza pública en los centros educativos para hacer efectiva la autonomía escolar. Desde el lado de las familias, las nuevas demandas surgidas tras la pandemia deben activarse y canalizarse hacia una participación que tenga el derecho a la educación como pilar central. Existe una oportunidad clara de aprovechar los cambios en la percepción pública sobre la educación y con ello de posicionarla más firmemente como política de Estado en cada país y, más allá, en cada región mundial.

Por último, la crisis del COVID es también la de la disponibilidad de datos fiables en el momento en el que de verdad hacen falta. Los poderes públicos han tenido muchas dificultades para comprender de manera precisa y detallada la evolución de la epidemia y sus consecuencias sanitarias y sociales. En el ámbito educativo, los sistemas de información y generación de conocimiento apenas se están desarrollando en la mayor parte de los países. Cabe preguntarse de qué manera es posible disponer de sistemas de información avanzados que permitan potenciar una agenda de investigación educativa que identifique y diagnostique las necesidades del sector y proporcione a sus distintos actores la evidencia empírica - y en tiempo real cuando se hace frente a una crisis como la del coronavirus - que permita tomar decisiones informadas y debatir más allá de las trincheras ideológicas.

\section{Referencias bibliográficas}

Andrew, A., Cattan, S., Costa-Dias, M., Farquharson, C.; Kraftman, L., Krutikova, S., Phimister, A., and Sevilla, A. (2020). Learning during the lockdown: real-time data on children's experiences during home learning. Cambridge: IFS. Disponible en https://www.ifs.org.uk/uploads/Edited_FinalBN288\%20Learning\%20during\%20the\%20lockdown.pdf

Banco Mundial (2020). The COVID-19 pandemic: shocks to education and policy responses. Washington DC: Banco Mundial, Working Paper. Disponible en https: / / openknowledge.worldbank.org/bitstream/handle/10986/33696/14819 8SP.pdf?sequence=6\&isAllowed $=y$

Bol, T. (2020). Inequality in homeschooling during the Corona crisis in the Netherlands. First results from the LISS Panel. doi: https://doi.org/10.31235/osf.io/hf32q 
Bonal, X. y Gonzalez, S. (2020). Desigualdades de Aprendizaje en Confinamiento. Resultados Destacados. https: / / blogs.uab.cat/aprenentatgeiconfinament

Bruns, B., Filmer, D., \& Patrinos, H. A. (2011). Making schools work: New evidence on accountability reforms. The World Bank, Washington DC. Disponible en http: //documents.worldbank.org/curated/en/483221468147581570/pdf/6005 00PUB0ID181s0Work09780821386798.pdf

Martínez García, J.S. y Molina Derteano, P. (2019). Fracaso escolar, crisis económica y desigualdad de oportunidades educativas: España y Argentina. Papers: Revista de Sociología 104(2), 270-303.

Moreno, J.M y Gortazar, L: (2020). Preparación de las escuelas para el aprendizaje digital, en opinión de los directores. Un análisis de PISA 2018 y sus implicancias para la respuesta a la crisis del COVID-19 (Coronavirus). Disponible en https: / /blogs.worldbank.org/es/education/schools-readiness-digital-learningeyes-principals-analysis-pisa-2018-and-its

Moreno, J.M. (2020). El día en que cerraron las escuelas de todo el mundo. En Luengo, F. y Gomez, J.A. (Coords.) Escenarios de Innovación Covid-19. La vuelta a la normalidad (pp. 7-12) Madrid: IOS. . Disponible en http://www.proyectoatlantida.eu/wordpress/wpcontent/uploads/2020/06/3Escenarios-investigaEDUCOVID.pdf

Pritchett, L. (2020). Developing Country schools need to reopen with different teaching. RISE Programme. Disponible en https: / / riseprogramme.org/blog/developing-country-schools-reopen

\section{Cómo citar este artículo:}

Moreno, J.M. \& Gortazar, L. (2020). Escolarización en Confinamiento: Experimento natural y prueba de esfuerzo. Revista de Currículum y Formación de Profesorado, 24(2), 168-181. DOI: 10.30827/profesorado.v24i2.15540 